\title{
PROJETO DE CORREIAS TRANSPORTADORAS: UM ESTUDO COMPUTACIONAL DE COMPARAÇÃO DOS MÉTODOS CEMA E PRÁTICO
}

\author{
D. A. SANTOS ${ }^{1}$ e R. A. MALAGONI ${ }^{1}$ \\ ${ }^{1}$ Faculdade de Engenharia Química-Universidade Federal de Uberlândia \\ dyrneq@yahoo.com.br-malagoni@feq.ufu.br
}

Artigo submetido em novembro/2013 e aceito em dezembro/2013

http://dx.doi.org/10.15628/holos.2014.1780

\section{RESUMO}

Para o projeto de correias transportadoras, são comumente empregados dois métodos: Método Prático e Método CEMA (Conveyor Equipment Manufacture Association). O Método Prático, mais simplificado, aplicase a transportadores simples e de pequena capacidade. Neste, calcula-se primeiramente a potência necessária para o transporte e, a partir desta, as tensões na correia. O método CEMA, mais sofisticado, é aplicável a transportadores de vários lances, curtos ou longos, onde calcula-se inicialmente as tensões em cada lance da correia, e, após isto, calcula-se a potência de acionamento. Com isto, um dos objetivos do presente trabalho foi a criação de um programa computacional, o qual realiza o projeto de correias transportadoras através dos métodos Prático e CEMA. Por meio deste programa, foram realizadas simulações para os materiais: rocha fosfática e minério de ferro (itabiritos) para diferentes configurações de correias transportadoras. Como dados de saída do programa, tem-se as seguintes especificações de projeto: largura da correia, distância do material à borda da correia, capacidade mássica, velocidade da correia e potência do motor. Tanto o Método Prático quanto o Método CEMA se mostraram de suma importância para o projeto de correias transportadoras, sendo o primeiro de grande valia na estimativa inicial de um projeto, enquanto o outro, apesar da maior complexidade, é o mais recomendado para o projeto final de correias transportadoras.

PALAVRAS-CHAVE: correia transportadora, método cema, método prático.

\section{CONVEYOR BELT DESIGN: A COMPUTATIONAL STUDY OF COMPARISON BETWEEN PRACTICAL AND CEMA METHODS}

\begin{abstract}
There are two main methods used for designing belt conveyors: Practical and CEMA (Conveyor Equipment Manufacture Association). The Practical Method is applicable for systems which convey materials at low to moderate capacities over medium distances. This method first determines the power needed for the drive and finally the stress distribution along the belt conveyor. On the other hand, the CEMA Method, considered more accurate than the Practical Method, first determines the stress distribution before the determination of the power needed for the drive. Higher capacity can be handled by
\end{abstract}

this method and it is applicable to more complex systems. In this work, the two methods cited above were implemented in a computer program which was used to carry out a comparison study taking into account the materials: phosphate rock and itabirite iron ore for different belt conveyor configurations. Both methods have proven to be very useful for designing belt conveyors. The Practical method is suitable for the initial design estimate while the CEMA method is the most recommended for the final design.

KEYWORDS: belt conveyor, cema method, practical method. 


\section{INTRODUÇÃO}

As correias transportadoras detêm uma posição dominante no transporte de materiais devido às suas inerentes vantagens, como economia e segurança de operação, confiabilidade, versatilidade e enorme gama de capacidades. Utilizadas nos mais diversos segmentos de mercado, as correias transportadoras possuem características técnicas que permitem sua aplicação em sistemas de transporte e elevação de materiais de pequeno, médio e grande porte, dependendo de sua adequada configuração (ZHANG e XIA, 2011; FERDORKO et al., 2013; ANDRIANOV e HORSSEN, 2008).

No setor minero-metalúrgico, observa-se que as correias transportadoras constituem o meio mais difundido de transporte para grandes quantidades de materiais a granel, reduzindo desta forma, a quantidade de caminhões e o custo deste tipo de serviço.

Em adição, as correias transportadoras são utilizadas em numerosos processos, em conexão com seu propósito normal de providenciar um fluxo contínuo de materiais entre operações. O sistema é basicamente formado por uma correia sem fim, a qual é estendida entre dois tambores (acionamento e de retorno) principais e roletes justapostos, sobre os quais a correia desliza, com baixo atrito, possibilitando a movimentação de cargas pesadas (FERDOKO e IVANCO, 2012).

O sistema possui alguns fatores limitantes como a velocidade do transporte e a inclinação da correia. A velocidade da correia depende do material a ser transportado e a inclinação da correia pode ser usada, segundo alguns autores, entre $12^{\circ}$ e $15^{\circ}$ em relação ao plano horizontal. Recentemente, a conformidade com os requerimento ambientais incentivou a seleção de correias transportadoras sobre outros meios de transporte (VANAMANE et al., 2011; YOU e QING, 2008).

Baseando-se neste contexto e no custo energético empregado no processamento de minérios, torna-se de suma importância o desenvolvimento e a aplicação de métodos cada vez mais precisos para o projeto de correias transportadoras. Um mau projeto destes equipamentos pode causar, dentre outros danos, o acúmulo de sujeiras nas suas lonas que podem se espalhar ao longo do transportador, acumulando-se em roletes de retorno e entradas de "shoot" de descarga. Tudo isso provoca acidentes e travamentos da correia e uma parada imprevista para manutenção acarreta prejuízos na linha de produção (CHEN et al., 2012; ZANOELO et al., 2008).

Para o projeto de correias transportadoras, são comumente empregados dois métodos: Método Prático e Método CEMA. O Método Prático, mais simplificado, aplica-se a transportadores simples e de pequena capacidade. Neste, calcula-se primeiramente a potência necessária para o transporte e, a partir desta, as tensões na correia. O método CEMA, mais sofisticado, é aplicável a transportadores de vários lances, curtos ou longos, onde calcula-se inicialmente as tensões em cada lance da correia, e, após isto, calcula-se a potência de acionamento (SWINDERMAN, 2007).

Com base no exposto acima, o objetivo do presente trabalho foi a confecção de um programa computacional, o qual realiza o projeto de correias transportadoras através dos métodos Prático e CEMA. Por meio deste programa, foram realizadas simulações com diferentes minérios e configurações de correias transportadoras. Como dados de saída do programa tem-se as seguintes 
especificações de projeto: largura da correia, distância do material à borda da correia, capacidade volumétrica, capacidade mássica, velocidade da correia e potência do motor.

\section{MATERIAL E MÉTODOS}

Para a confecção do programa computacional que realiza o projeto de correias transportadoras utilizou-se da ferramenta matemática Scilab versão 4.1.2. Embora o programa aqui desenvolvido contenha um banco de dados incluindo diversos tipos de minérios, o presente trabalho irá considerar, a fim de exemplificação, apenas dois diferentes materiais: rocha fosfática e minério de ferro (itabiritos) com massas específicas de 2980 e $3580 \mathrm{~kg} / \mathrm{m}^{3}$, respectivamente.

Os métodos utilizados para o projeto de correias transportadoras foram: Método Prático e Método CEMA. Antes de detalhar cada um dos métodos citados anteriormente, vale ressaltar que alguns parâmetros importantes de entrada no projeto de correias transportadoras são os mesmos para os dois métodos, pois dependem, basicamente, das características dos materiais a serem transportados (VANAMANE e MANE, 2012).

A capacidade $(C)$ de um transportador é função da área de sua seção transversal, da velocidade da correia $(V)$ e do peso específico do material. A área da secção transversal é função da largura da correia, do número e inclinação $(\beta)$ dos roletes e do ângulo de acomodação do material na correia. $O$ ângulo de acomodação $(\alpha)$ é uma característica do material em movimento na correia, ocorrendo devido à tendência de nivelamento do material causada pela trepidação da correia nos roletes. A Tabela 1 fornece, de forma resumida, as capacidades volumétricas de um transportador horizontal a uma velocidade de $1,0 \mathrm{~m} / \mathrm{s}$.

Tabela 1 - Capacidades volumétricas de um transportador horizontal a uma velocidade de 1,0 m/s.

\begin{tabular}{|c|c|c|c|c|c|c|}
\hline \multirow{3}{*}{ Roletes } & \multirow{3}{*}{$\begin{array}{c}\text { Ângulo de } \\
\text { Acomodação }(\alpha)\end{array}$} & \multicolumn{5}{|c|}{ Capacidade Volumétrica $\left(C_{t a b .}\right)\left(\mathrm{m}^{3} / \mathrm{h}\right)$ a $1,0 \mathrm{~m} / \mathrm{s}$} \\
\hline & & \multicolumn{5}{|c|}{ Largura da correia (m) } \\
\hline & & 0,41 & 0,51 & 1,1 & 1,5 & 2,1 \\
\hline \multirow{3}{*}{$\begin{array}{c}3 \text { roletes iguais } \\
\beta=35^{\circ}\end{array}$} & $10^{\circ}$ & $\ldots$ & $\ldots$ & 384 & 812 & 1631 \\
\hline & $20^{\circ}$ & $\ldots$ & $\ldots$ & 455 & 961 & 1929 \\
\hline & $30^{\circ}$ & $\cdots$ & $\ldots$ & 530 & 1118 & 2242 \\
\hline \multirow{3}{*}{$\begin{array}{c}3 \text { roletes iguais } \\
\beta=45^{\circ}\end{array}$} & $10^{\circ}$ & $\ldots$ & $\ldots$ & 430 & 909 & 1822 \\
\hline & $20^{\circ}$ & $\ldots$ & $\ldots$ & 492 & 1038 & 2079 \\
\hline & $30^{\circ}$ & $\ldots$ & $\ldots$ & 556 & 1173 & 2349 \\
\hline
\end{tabular}

Após a obtenção da capacidade volumétrica tabelada (Ctab.), deve-se realizar, através de fatores de correção, o cálculo da capacidade volumétrica real a uma determinada velocidade desejada (V) e a uma determinada inclinação do transportador $(\lambda)$ (Equação 1). Sendo o valor do fator de correção ilustrado na Tabela 2.

$$
C=C_{T a b} V K
$$

equação (1) 
sendo: C, $C_{T a b}, \mathrm{~V}$ e K, a capacidade volumétrica real, a capacidade volumétrica tabelada, a velocidade desejada do transportador e o fator de correção da capacidade de um transportador, respectivamente.

Tabela 2 - Fator de correção para capacidade volumétrica real.

\begin{tabular}{c|c|c|c|c|c|c|c}
\hline $\boldsymbol{\lambda}$ & $0^{\circ}$ & $6^{\circ}$ & $10^{\circ}$ & $14^{\circ}$ & $18^{\circ}$ & $21^{\circ}$ & $23^{\circ}$ \\
\hline $\boldsymbol{K}$ & 1 & 0,98 & 0,95 & 0,91 & 0,85 & 0,78 & 0,73 \\
\hline
\end{tabular}

A Tabela 3 mostra, de forma resumida, as velocidades máximas do transportador, as quais dependem das características dos materiais transportados. Para material seco e fino, uma velocidade elevada pode causar muita poeira. Para material pesado de grande granulometria ou com partículas pontiagudas, uma velocidade elevada pode causar muito desgaste nas calhas de descarga.

Tabela 3 - Velocidade máxima recomendada $(\mathrm{m} / \mathrm{s})$.

\begin{tabular}{c|c|c|c}
\hline $\begin{array}{c}\text { Largura da } \\
\text { Correia }(\mathrm{m})\end{array}$ & $\begin{array}{c}\text { Cereais e outros } \\
\text { materiais de escoamento } \\
\text { fácil e não abrasivos }\end{array}$ & $\begin{array}{c}\text { Carvão, terra, minérios } \\
\text { desagregados, pedra britada } \\
\text { fina e pouco abrasiva }\end{array}$ & $\begin{array}{c}\text { Minérios e pedras duras, } \\
\text { pontiagudos pesados e } \\
\text { muito abrasivos }\end{array}$ \\
\hline 0,41 & 2,5 & 1,6 & 1,6 \\
\hline 0,61 & 3,0 & 2,5 & 2,3 \\
\hline 0,91 & 4,1 & 3,3 & 3,0 \\
\hline 1,10 & 4,1 & 3,6 & 3,0 \\
\hline
\end{tabular}

A seleção da largura da correia é determinada simultaneamente pela capacidade volumétrica desejada (C) e pela porcentagem de tamanho máximo do material (granulometria) (Tabela 4).

Tabela 4 - Largura da correia em função da granulometria do material transportado.

\begin{tabular}{c|c|c|c|c|c|c}
\hline \multirow{2}{*}{$\begin{array}{c}\text { Largura da } \\
\begin{array}{c}\text { Correia } \\
(\mathrm{m})\end{array}\end{array}$} & \multicolumn{2}{|c|}{$\alpha=10^{\circ}$} & \multicolumn{2}{c}{$\alpha=20^{\circ}$} & \multicolumn{3}{c}{$\alpha=30^{\circ}$} \\
\cline { 2 - 7 } & $\begin{array}{c}90 \% \text { pedaços } \\
\text { e } \\
10 \% \text { finos }\end{array}$ & $\begin{array}{c}100 \% \\
\text { pedaços }\end{array}$ & $\begin{array}{c}90 \% \text { pedaços } \\
\text { e } \\
10 \% \text { finos }\end{array}$ & $\begin{array}{c}\text { Tamanho Máximo do Material (m) } \\
\text { pedaços }\end{array}$ & $\begin{array}{c}90 \% \text { pedaços } \\
\text { e } \\
10 \% \text { finos }\end{array}$ & $\begin{array}{c}\text { 100\% } \\
\text { pedaços }\end{array}$ \\
\hline 0,41 & 0,20 & 0,13 & 0,13 & 0,08 & 0,07 & 0,04 \\
\hline 0,61 & 0,30 & 0,20 & 0,20 & 0,12 & 0,10 & 0,06 \\
\hline 0,91 & 0,46 & 0,30 & 0,30 & 0,18 & 0,15 & 0,09 \\
\hline 1,22 & 0,61 & 0,41 & 0,41 & 0,24 & 0,20 & 0,12 \\
\hline 1,37 & 0,69 & 0,46 & 0,46 & 0,27 & 0,23 & 0,14 \\
\hline
\end{tabular}

\subsection{Cálculo da potência de acionamento}

O acionamento da correia é feito por um único tambor (acionamento simples) ou por dois tambores (acionamento duplo). A potência de um transportador é composta de quatro grandes parcelas: 
- A necessária para vencer as forças de inércia dos roletes, tambores e correia, isto é, para movimentar o transportador vazio;

- A necessária para o deslocamento horizontal do material;

- A necessária para o deslocamento vertical do material, existente nos transportadores em aclive ou declive;

- A necessária para vencer o atrito de acessórios, tais como raspadores, limpadores, guias laterais, etc.

Desta forma serão apresentados, a seguir, os dois métodos para o cálculo da potência do transportador de correias: Método Prático e Método CEMA.

\subsubsection{Método Prático}

Para o método Prático, calcula-se primeiramente a potência necessária para o transporte e, a partir desta, as tensões na correia. A potência efetiva necessária para o transporte do material é calculada pela Equação 2 .

$$
N_{E}=\frac{V}{1}\left(N_{V}+N_{G}\right)+(Q / 27,8)\left(N_{1} \pm N_{h}\right)
$$

equação (2)

sendo: $N_{E}, V, N_{V}, N_{G}, Q, N_{1}$ e $N_{h}$, a potência total efetiva, a velocidade de projeto, a potência para acionar o transportador vazio a um a velocidade de 1,0 m/s, a potência para vencer o atrito das guias laterais à velocidade de $1,0 \mathrm{~m} / \mathrm{s}$, a capacidade mássica, a potência para deslocar $27,8 \mathrm{~kg} / \mathrm{s}$ de material de uma distância na horizontal e a potência para elevar ou descer $27,8 \mathrm{~kg} / \mathrm{s}$ de material de certa altura, respectivamente.

Com a potência efetiva $\left(N_{E}\right)$, pode-se obter a tensão efetiva na correia $\left(T_{e}\right)$, que é a força tangencial que movimenta a correia (Equação 3).

$$
T_{e}=\frac{75 N_{E}}{V}
$$

equação (3)

Os parâmetros $N_{V}, N_{1}, N_{h}$ e $N_{G}$, utilizados na Equação 2, são dados, de forma resumida, pelas tabelas 5, 6, 7 e 8, respectivamente.

\begin{tabular}{|c|c|c|c|c|c|c|c|c|c|c|c|c|c|}
\hline \multirow{2}{*}{$\begin{array}{l}\text { Largura da } \\
\text { correia }(\mathrm{m})\end{array}$} & \multicolumn{13}{|c|}{ Comprimento total do transportador $(\mathrm{m})$} \\
\hline & 10 & 15 & 20 & 25 & 30 & 40 & 50 & 60 & 70 & 80 & 90 & 100 & 110 \\
\hline 0,41 & 0,37 & 0,47 & 0,54 & 0,61 & 0,7 & 0,8 & 0,9 & 1,01 & 1,1 & 1,2 & 1,31 & 1,42 & 1,53 \\
\hline 0,51 & 0,45 & 0,55 & 0,64 & 0,72 & 0,81 & 0,95 & 1,09 & 1,2 & 1,32 & 1,42 & 1,54 & 1,67 & 1,8 \\
\hline
\end{tabular}

Tabela 5 - Potência $N_{V}(h p)$. 


\begin{tabular}{c|c|c|c|c|c|c|c|c|c|c|c|c|c}
\hline 0,61 & 0,57 & 0,7 & 0,83 & 0,91 & 1,01 & 1,2 & 1,33 & 1,52 & 1,67 & 1,8 & 1,92 & 2,06 & 2,19 \\
\hline 0,76 & 0,69 & 0,81 & 0,97 & 1,1 & 1,22 & 1,44 & 1,66 & 1,83 & 2,04 & 2,19 & 2,39 & 2,55 & 2,71 \\
\hline
\end{tabular}

Tabela 6 - Potência $N_{1}(h p)$.

\begin{tabular}{c|c|c|c|c|c|c|c|c|c|c|c|c|c}
\hline $\begin{array}{c}\text { Distância na } \\
\text { horizontal }(\mathrm{m})\end{array}$ & 10 & 15 & 20 & 25 & 30 & 40 & 50 & 60 & 70 & 80 & 90 & 100 & 110 \\
\hline$N_{1}(h p)$ & 0,5 & 0,6 & 0,74 & 0,81 & 0,95 & 1,1 & 1,25 & 1,4 & 1,5 & 1,64 & 1,75 & 1,87 & 2,1 \\
\hline
\end{tabular}

Tabela 7 - Potência $N_{h}(h p)$.

\begin{tabular}{c|c|c|c|c|c|c|c|c|c|c|c|c|c}
\hline Distância na vertical $(\mathrm{m})$ & 2 & 3 & 5 & 7,5 & 10 & 12,5 & 15 & 17,5 & 20 & 22,5 & 25 & 27,5 & 30 \\
\hline$N_{h}(h p)$ & 0,8 & 1,2 & 1,9 & 2,8 & 3,7 & 4,7 & 5,6 & 6,5 & 7,4 & 8,4 & 9,3 & 10,2 & 11,1 \\
\hline
\end{tabular}

Tabela 8 - Potência $\mathrm{N}_{\mathrm{G}}(\boldsymbol{h p})$.

\begin{tabular}{c|c|c|c|c|c|c|c|c|c|c|c|c|c}
\hline $\begin{array}{c}\text { Comprimento das } \\
\text { guias laterais (m) }\end{array}$ & 5 & 10 & 20 & 25 & 30 & 35 & 40 & 45 & 50 & 55 & 60 & 65 & 70 \\
\hline$N_{G}(h p)$ & 0,6 & 1,26 & 2,52 & 3,18 & 3,84 & 4,56 & 5,28 & 6,0 & 6,72 & 7,4 & 8,1 & 8,9 & 9,6 \\
\hline
\end{tabular}

\subsubsection{Método CEMA}

Para o método CEMA (SWINDERMAN, 2007), calculam-se inicialmente as tensões em cada lance da correia através da Equação 4.

$$
T_{e}=L\left(K_{X}+K_{Y} W_{b}+0,015 W_{b}\right) \pm H W_{m}+T_{a}
$$

equação (4)

sendo: $T_{e}, L, K_{X}, K_{y}, W_{b}, H, W_{m}$ e $T_{a}$, a tensão efetiva na correia, o comprimento do transportador, a resistência à rotação dos roletes e ao deslizamento da correia sobre os mesmos, o fator relativo às resistências à flexão da correia e do material sobre os roletes, o peso linear da correia, a altura de elevação ou descida do material na correia (sendo positivo para subida e negativa para descida), o peso do material na correia e a tensão para vencer o atrito dos acessórios e para acelerar o material, respectivamente.

Obtida a tensão efetiva $\left(T_{e}\right)$ determina-se a potência efetiva $\left(N_{e}\right)$ necessária para o transporte do material, pela Equação 5.

$$
N_{e}=\frac{T_{e} V}{75}
$$

A tensão para vencer o atrito dos acessórios e acelerar o material é dada, de forma resumida, através da soma das parcelas indicadas abaixo (Equação 6). 
$T_{a}=F_{g}+F_{d}+F_{1}+F_{a}$

equação (6)

Cujos parâmetros são calculados da seguinte forma:

- $\quad$ Parâmetro $F_{g}$ - é a força necessária para vencer o atrito do material com as guias laterais (Equação 7).

$F_{g}=0,01488 C_{S} L_{g} B^{2}+8,92 L_{g}$ equação (7)

sendo: $F_{g}, L_{g}, B$ e $C_{S}$, a força, o comprimento das guias laterais, a largura da correia e o fator devido ao atrito do material com as guias (Tabela 9), respectivamente.

Tabela 9 - Fator $C_{s}$, utilizado na Equação 7, para alguns materiais.

\begin{tabular}{c|c}
\hline$C_{S}$ & Material \\
\hline 0,1378 & Areia seca de aterro \\
\hline 0,1881 & Bauxita, resíduos \\
\hline 0,1086 & Rocha fosfática \\
\hline 0,2760 & Minério de ferro \\
\hline
\end{tabular}

- $\quad$ Parâmetro $F_{d}$ - é a força necessária para vencer o atrito dos desviadores. Para $100 \%$ de material desviado, o valor de $F_{d}$ (dado em $\mathrm{kgf}$ ) é de 0,55 vezes a largura da correia $(B)$ dada em polegadas (in), enquanto para $50 \%$ de material desviado, o valor de $F_{d}$ é de 0,36 vezes a largura da correia.

- $\quad$ Parâmetro $F_{1}$ - é a força necessária para vencer o atrito dos raspadores e limpadores, sendo seu valor (dado em $\mathrm{kgf}$ ) de 1,15 vezes a largura da correia (B) em polegadas (in), para cada raspador ou limpador.

- $\quad$ Parâmetro $F_{a}$ - força necessária para acelerar o material (Equação 8).

$$
F_{a}=\frac{Q\left(V^{2}-V_{C}^{2}\right)}{36 V}
$$

sendo: $V_{c}$ a componente da velocidade do material na direção do deslocamento da correia.

Para o cálculo de $K_{x}$ (Equação 10), resistência à rotação dos roletes e ao deslizamento da correia sobre os mesmos, utilizado na Equação 4, deve-se, primeiro, determinar o peso linear da correia (Tabela 10). 
Tabela 10 - Valores médios para peso linear da correia $W_{b}(\mathrm{~kg} / \mathrm{m})$.

\begin{tabular}{c|c|c|c}
\hline \multirow{2}{*}{ Largura da Correia $(\mathrm{m})$} & \multicolumn{3}{|c}{ Peso Específico do Material $\left(\mathrm{kg} / \mathrm{m}^{3}\right)$} \\
\cline { 2 - 4 } & $570-1410$ & $860-2450$ & $2470-3800$ \\
\hline 0,46 & 5,2 & 6,0 & 6,7 \\
0,61 & 6,7 & 8,2 & 9,0 \\
0,76 & 9,0 & 10,4 & 12,0 \\
\hline 0,91 & 13,4 & 15,0 & 17,86 \\
1,07 & 16,4 & 17,86 & 21,0 \\
1,22 & 20,8 & 22,3 & 25,3 \\
\hline 2,13 & 37,2 & 44,6 & 49,1 \\
2,44 & 44,6 & 52,1 & 56,6 \\
\hline
\end{tabular}

Os valores acima devem ser considerados como primeira aproximação sendo que, após conhecida a correia, seu peso deverá ser recalculado. O peso linear do material $\left(W_{m}\right)$ é dado pela Equação 9.

$$
W_{m}=0,277 \frac{Q}{V}
$$

Logo:

$$
K_{X}=0,00068(W m+W b)+\frac{X}{a}
$$

sendo: $X$ e $a$ o coeficiente função dos diâmetros do eixo e do rolo dos roletes (Tabela 11) e o espaçamento entre os roletes, respectivamente.

Tabela 11 - Valores do coeficiente $X$.

\begin{tabular}{c|c|c}
\hline$x$ & Diâmetro dos rolos $(\mathrm{m})$ & Série dos rolos \\
\hline 1,5 & 0,15 & CEMA C6, D6 \\
\hline 1,8 & 0,13 & CEMA B5, C5, D5 \\
\hline 2,3 & 0,10 & CEMA B4, C4 \\
\hline 2,4 & 0,18 & CEMA E7 \\
\hline 2,8 & 0,15 & CEMA E6 \\
\hline
\end{tabular}

Finalmente, o último parâmetro necessário ao cálculo do método CEMA, ou seja, $K_{Y}$, fator relativo às resistências à flexão da correia e do material sobre os roletes, pode ser extraído da Tabela 12, resumida a seguir.

Tabela 12 - Valores do parâmetro $K_{y}$.

\begin{tabular}{c|c|c|c|c|c|c|c|c}
\hline \multirow{2}{*}{$\begin{array}{c}\text { Comprimento total do } \\
\text { transportador } L(m)\end{array}$} & \multirow{2}{*}{$\begin{array}{c}W_{b}+W_{m} \\
(\mathrm{~kg} / \mathrm{m})\end{array}$} & \multicolumn{6}{|c}{ Ângulo de inclinação da correia $\left({ }^{\circ}\right)$} \\
\cline { 3 - 9 } & & 0 & 2 & 3,5 & 5 & 7 & 14 & 18 \\
\hline \multirow{2}{*}{76} & 30 & 0,035 & 0,035 & 0,034 & 0,031 & 0,031 & 0,031 & 0,031 \\
& 74,4 & 0,035 & 0,034 & 0,033 & 0,032 & 0,031 & 0,028 & 0,027 \\
& 111,6 & 0,035 & 0,034 & 0,032 & 0,032 & 0,03 & 0,027 & 0,025 \\
\hline
\end{tabular}




\begin{tabular}{|c|c|c|c|c|c|c|c|c|}
\hline & 149 & 0,035 & 0,033 & 0,032 & 0,031 & 0,03 & 0,026 & 0,023 \\
\hline & 223,2 & 0,035 & 0,035 & 0,034 & 0,033 & 0,031 & 0,025 & 0,021 \\
\hline & 297,6 & 0,035 & 0,035 & 0,035 & 0,035 & 0,032 & 0,024 & 0,018 \\
\hline & 372 & 0,035 & 0,035 & 0,035 & 0,035 & 0,033 & 0,021 & 0,018 \\
\hline & 446,4 & 0,035 & 0,035 & 0,035 & 0,035 & 0,032 & 0,019 & 0,018 \\
\hline \multirow{8}{*}{244} & 30 & 0,035 & 0,031 & 0,03 & 0,029 & 0,029 & 0,029 & 0,029 \\
\hline & 74,4 & 0,032 & 0,029 & 0,028 & 0,026 & 0,025 & 0,021 & 0,018 \\
\hline & 111,6 & 0,031 & 0,029 & 0,026 & 0,024 & 0,022 & 0,016 & 0,016 \\
\hline & 149 & 0,031 & 0,028 & 0,025 & 0,022 & 0,02 & 0,016 & 0,016 \\
\hline & 223,2 & 0,034 & 0,028 & 0,023 & 0,019 & 0,017 & 0,016 & 0,016 \\
\hline & 297,6 & 0,035 & 0,027 & 0,021 & 0,016 & 0,016 & 0,016 & 0,016 \\
\hline & 372 & 0,035 & 0,026 & 0,02 & 0,017 & 0,016 & 0,016 & 0,016 \\
\hline & 446,4 & 0,035 & 0,025 & 0,018 & 0,018 & 0,018 & 0,018 & 0,018 \\
\hline \multirow{7}{*}{427} & 74,0 & 0,029 & 0,026 & 0,024 & 0,022 & 0,021 & 0,016 & 0,016 \\
\hline & 111,6 & 0,028 & 0,024 & 0,021 & 0,019 & 0,016 & 0,016 & 0,016 \\
\hline & 149 & 0,028 & 0,023 & 0,019 & 0,016 & 0,016 & 0,016 & 0,016 \\
\hline & 223,2 & 0,029 & 0,02 & 0,016 & 0,016 & 0,016 & 0,016 & 0,016 \\
\hline & 297,6 & 0,03 & 0,021 & 0,016 & 0,016 & 0,016 & 0,016 & 0,016 \\
\hline & 372 & 0,03 & 0,02 & 0,017 & 0,016 & 0,016 & 0,016 & 0,016 \\
\hline & 446,4 & 0,03 & 0,019 & 0,018 & 0,018 & 0,018 & 0,018 & 0,018 \\
\hline
\end{tabular}

\section{RESULTADOS E DISCUSSÃO}

Para o projeto de correias transportadoras utilizou-se de duas configurações diferentes: Configuração 1 e Configuração 2 (Tabela 13).

Tabela 13 - Configurações utilizadas para o projeto de correias transportadoras.

\begin{tabular}{c|c|c}
\hline Parâmetros & Configuração 1 & Configuração2 \\
\hline Material transportado & Rocha fosfática & Minério de ferro (itabiritos) \\
\hline Granulometria & $90 \%$ de pedaços e 10\% de & finos \\
\hline Tamanho máximo $(\mathrm{m})$ & 0,2 & 0,25 \\
\hline Ângulo de acomodação $\left(^{\circ}\right)$ & 20 & 20 \\
\hline Número de roletes & 3 & 3 \\
\hline Inclinação dos roletes $\left({ }^{\circ}\right)$ & 35 & 45 \\
\hline Velocidade projeto $(\mathrm{m} / \mathrm{s})$ & $80 \%$ da velocidade máxima & $85 \%$ da velocidade máxima \\
\hline Peso específico $\left(\mathrm{kg} / \mathrm{m}^{3}\right)$ & 2980 & 3580 \\
\hline Numero de guias laterais & 2 & 3 \\
\hline Comprimento das guias laterais $(\mathrm{m})$ & 30 & 25 \\
\hline Distância na horizontal $(\mathrm{m})$ & 90 & 120 \\
\hline Distância na vertical $(\mathrm{m})$ & 12 & 18 \\
\hline Capacidade de projeto $(\mathrm{kg} / \mathrm{s})$ & $90 \%$ da capacidade máxima & $92 \%$ da capacidade máxima \\
\hline
\end{tabular}




\begin{tabular}{c|c|c}
\hline Classe dos roletes & Classe E6 & Classe E6 \\
\hline Espaçamento entre os roletes $(\mathrm{m})$ & 1,1 & 1,1 \\
\hline Tipo de esticador & Esticador de gravidade & Esticador de gravidade \\
\hline Revestimento do tambor & Borracha & Borracha \\
\hline
\end{tabular}

Os resultados dos projetos utilizando-se as configurações 1 e 2 foram dispostos na Figura 1. O projeto utilizando o método CEMA foi dividido em duas categorias: Método CEMA com simples acionamento (CEMA-SA) e Método CEMA com duplo acionamento (CEMA-DA), no intuito de avaliar, também, a influência dos acessórios. Para o método Prático, a opção de duplo acionamento não é levada em consideração, logo, utilizou-se apenas o simples acionamento.

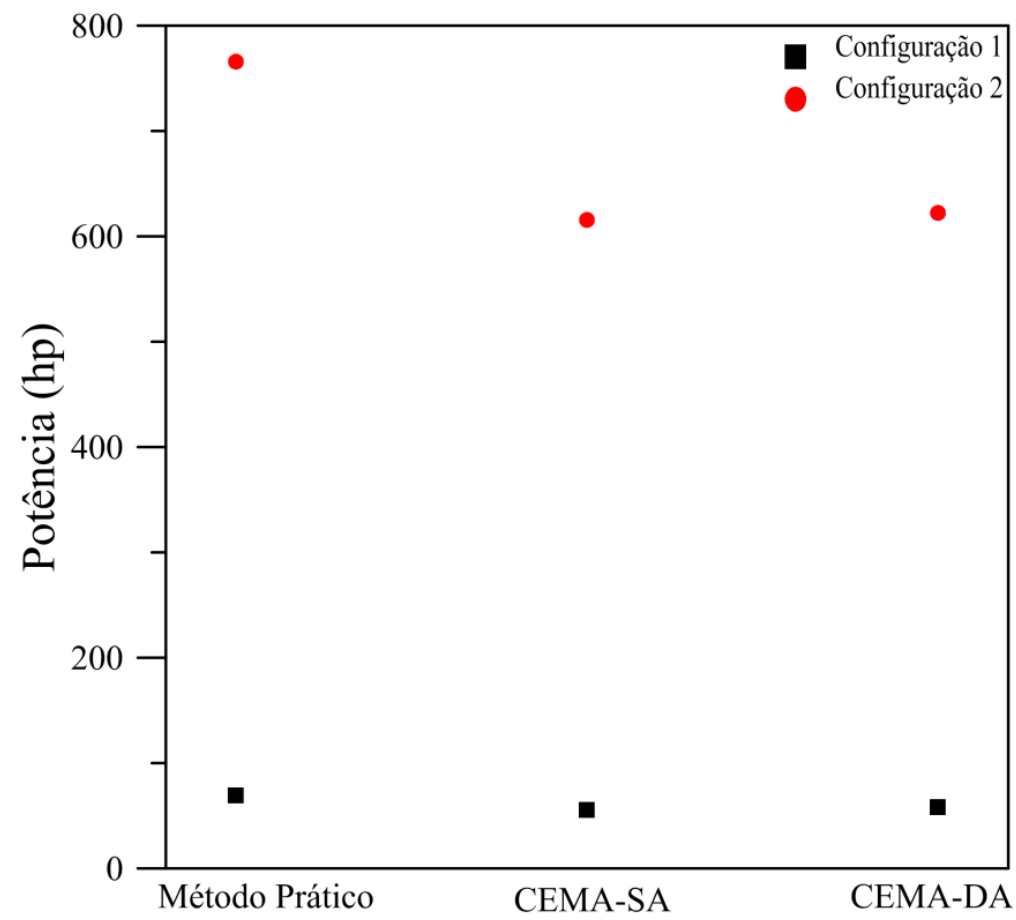

Figura 1 - Potência de acionamento em função dos métodos de projeto de correias transportadoras para diferentes configurações.

Observou-se pela Figura 1, que para a configuração 1 e 2, a potência do motor necessária para transportar o material calculada pelo método Prático, foi de aproximadamente $24,2 \%$ maior do que aquela calculada pelo método CEMA com simples acionamento (CEMA-SA). Para o método CEMA, a maior potência requerida foi quando se utilizou o duplo acionamento, o que já era de se esperar, pois quanto maior o número de acessórios, maior o atrito entre estes e a correia.

Os valores da largura da correia $(B)$, velocidade de projeto da correia $(V)$, capacidade mássica de projeto $(Q)$ e distância do material à borda da correia $\left(D_{m}\right)$ dependem somente das características dos materiais a serem transportados, logo, independe do método de projeto utilizado. Para a configuração 1, os valores calculados foram de 0,61 m, 1,85 m/s, 180,31 kg/s e $0,06 \mathrm{~m}$, respectivamente. Já para a configuração 2 , estes valores foram de 1,28 m, 2,81 m/s, $1782,84 \mathrm{~kg} / \mathrm{s}$ e $0,09 \mathrm{~m}$, respectivamente. 
Em relação aos materiais transportados e as diferentes configurações utilizadas, observouse que a configuração 2 necessitou de uma maior potência quando comparada à configuração 1 , devido aos seguintes fatores: a massa específica do minério de ferro é $20 \%$ maior do que a da rocha fosfática; a velocidade de projeto da configuração 2 foi maior do que a da configuração 1 , ocasionando uma maior capacidade mássica de transporte; a configuração 2 utilizou um número maior de guias laterais do que a configuração 1, ocasionando um maior atrito com a correia; tanto a distância na horizontal quanto na vertical foram maiores na configuração 2 , além do fato da eficiência do motor ser menor nesta configuração.

\section{CONCLUSÃO}

A partir dos resultados obtidos, pôde-se concluir que tanto o Método Prático quanto o Método CEMA se mostraram de suma importância para o projeto de correias transportadoras, sendo o primeiro de grande valia na estimativa inicial de um projeto, enquanto o outro, apesar da maior complexidade, é o mais recomendado para o projeto final de correias transportadoras. Vale ressaltar, também, a grande necessidade em se desenvolver métodos cada vez mais precisos para o projeto de correias transportadoras visando, dentre outros fatores, a maximização da economia energética e a minimização de materiais dispersos no ambiente.

\section{AGRADECIMENTOS}

Agradecemos a Fundação de Amparo à Pesquisa do Estado de Minas Gerais (FAPEMIG) pelos recursos concedidos no Projeto de Participação Coletiva em Evento Científico ou Tecnológico (PCE-00019-13).

\section{REFERÊNCIAS BIBLIOGRÁFICAS}

1. ANDRIANOV, I. V., HORSSEN, W. T. On the transversal vibrations of a conveyor belt: applicability of simplified models, Journal of Sound and Vibration, v. 313, p. 822-829, 2008.

2. CHEN, X.L., WHEELER, C.A., DONOHUE, T.J., MCLEAN, R., ROBERTS, A.W. Evaluation of dust emissions from conveyor transfer chutes using experimental and CFD simulation, International Journal of Mineral Processing, v. 110, p. 101-108, 2012.

3. FÁBRICA DE AÇO PAULISTA S. A. Manual de transportadores contínuos. 3a edição. 300 p. Brasil. 1981.

4. FEDORKO, G., IVANCO, V. Analysis of force ratios in conveyor belt of classic belt conveyor, Procedia Engineering, v. 48, p. $123-128,2012$.

5. FEDORKO, G., MOLNÁR, V., Z IVCÁK, J., DOVICA, M., HUSÁKOVÁ, N. Failure analysis of textile rubber conveyor belt damaged by dynamic wear, Engineering Failure Analysis, v. 28, p. 103114, 2013.

6. SWINDERMAN, R.T. CEMA (Conveyor Equipment Manufacturers Association) - Belt Conveyors for Bulk Materials, Chaners Publishing Company, Inc. 6 th edition, p. 567, 2007.

7. VANAMANE, S.S., MANE, P. A., INAMDAR K.H. Introduction to belt conveyor system used for cooling of mould, National Conference on Recent Advances in Manufacturing Engineering and 
Technology, Hyderabad, p. 104-108, 2011.

8. YOU, -F. H., QING, -R. M. Dynamic characteristics of conveyor belts, J. China Univ Mining \& Technol, v. 18, p. 0629-0633, 2008.

9. ZANOELO, E.F., ABITANTE, A., MELEIRO, L.A.C. Dynamic modeling and feedback control for conveyors-belt dryers of mate leaves, Journal of Food Engineering, v. 84, p. 458-468, 2008.

10. ZHANG, S., XIA, X. Modeling and energy efficiency optimization of belt conveyors, Applied Energy, v. 88, p. 3061-3071, 2011. 